Cómo citar este artículo: Espinel-Rubio, G. A., Hernández-Suárez, C. A., \& Rojas-Suárez, J. P. (2020). Las TIC como medio socio-relacional: un análisis descriptivo en el contexto escolar con adolescentes de educación media. Rev.investig.desarro.innov., 11 (1), 99-112.

\title{
Las TIC como medio socio-relacional: un análisis descriptivo en el contexto escolar con adolescentes de educación media
}

\section{ICT as a socio-relational medium: a descriptive analysis in the school context with high school adolescents}

\author{
Gladys Adriana Espinel-Rubio' \\ César Augusto Hernández-Suárez ${ }^{2}$ \\ Jhan Piero Rojas-Suárez ${ }^{3}$
}

Recibido: febrero 27 de 2020

Aceptado: junio 25 de 2020

\section{Resumen}

El propósito de esta investigación, fue identificar los nexos socio-relacionales del uso de las TIC en el entorno escolar de los adolescentes. La investigación tuvo un enfoque mixto, con una muestra de 40 adolescentes, con edades entre 14 y 17 años, pertenecientes a los grados 9 a 11 de una institución educativa colombiana. La información se recolectó a través del mapa socio-relacional, que consistió en graficar las relaciones e interacciones de la población objetivo, en los ámbitos virtual y presencial. Los resultados indican que el número de relaciones que tienen los adolescentes en el medio virtual, son diferentes a las del medio presencial. Esto se debe a que existe mayor conectividad comunicativa. Se concluye que las interacciones sociales mediadas por TIC, se presentan de dos formas: la interacción conectiva de costumbre, con los mismos sujetos del ámbito presencial, y la conectiva ampliada, que se da con individuos diferentes a los de costumbre.

Palabras clave: TIC, redes sociales, mapa socio-relacional, entorno escolar.

\begin{abstract}
The purpose of this research was to identify the socio-relational links of the use of ICTs in the adolescent school environment. The research had a mixed approach, with a sample of 40 adolescents, aged 14-17, from grades 9-11 in a Colombian educational institution. The information was collected through the socio-relational mapping, which consisted of graphing the relationships and interactions of the target population, in the virtual and face-to-face environments. The results indicate that the number of relationships that adolescents have in the virtual environment are different from those in the face-to-face environment. This is because there is a greater communicative connectivity. It is concluded that social interactions mediated by ICTs take two forms: the usual connective interaction with the same subjects in the face-to-face environment, and the extended connectivity, which occurs with individuals different from the usual ones.
\end{abstract}

Keywords: ICT, social networks, socio-relational map, school environment.

1 Comunicadora Social, Magíster en Estudios sobre Problemas Políticos Latinoamericanos, Universidad Francisco de Paula Santander, Cúcuta, Colombia. E-mail: gladysespinel@ufps.edu.co. ORCID: 0000-0002-8796-9257

2 Licenciado en Matemáticas y Computación, Magíster en Enseñanza de las Ciencias, Universidad Francisco de Paula Santander, Cúcuta, Colombia. E-mail: cesaraugusto@ufps.edu.co. ORCID: 0000-0002-7974-5560

3 Ingeniero Civil, Magíster en Ingeniería, Universidad Francisco de Paula Santander, Cúcuta, Colombia. E-mail: jhanpierorojas@ufps.edu.co. ORCID: 0000-0003-2682-9880 


\section{Introducción}

La educación es un área en la que se desarrollan con más fuerza las TIC. en Colombia, se asumen como un requisito fundamental para la calidad, el uso e implementación de nuevas plataformas tecnológicas. Este contexto favorece el hecho de que los niños se inicien en el uso del internet y las redes sociales a edades cada vez MÁS TEMPRANAS (Rueda-Rueda \& Rico-Bautista, 2016); sin embargo, las TIC en sus diferentes formas, tienen implicaciones dentro y fuera del ámbito escolar. Al respecto, el Departamento Administrativo Nacional de Estadística (DANE), señalan que en Colombia:

(...) el $60,6 \%$ de personas en el territorio nacional usa un computador, de ellos $59 \%$ se conecta a internet. Los principales usuarios de internet, según el rango de edad, son los jóvenes entre 12 y 24 años en $80,6 \%$, siendo los estudiantes de secundaria y universitarios los principales usuarios. (López-Ponce \& Arcila-Calderón, 2016, p. 291)

Esta realidad se refuerza por la incorporación de recurso hardware al contexto educativo. Esto obliga al sistema a someterse a una acelerada adaptación a los avances, implicando la aplicación de metodologías de formación adecuadas, en las que se promueva el equilibrio entre información, conocimiento, comunicación y producción del mismo conocimiento. En ese orden, las TIC están relacionadas con el contexto escolar en el sentido social (comunicación e interacción virtual), y de consulta (Islas-Torres, 2017).

Rueda-Rueda y Rico-Bautista (2016), revelan que"el $68 \%$ de los jóvenes entra todos los días a internet, el $32 \%$ se conecta entre tres o más horas al día y el $89 \%$ navega desde su computador personal o el celular". Entonces, es válido reconocer a las TIC como agente socializador, que inciden en la personalidad, especialmente, en las etapas de niñez y adolescencia, puesto que estas poseen influjo para: modificar, formar o transformar el pensar y el actuar de los individuos. En este sentido, Carrillo-David (2015), afirma que la "influencia que pueden tener las TIC en la transformación de los diferentes ámbitos socializadores (familia, escuela, grupo de amigos) y en las prácticas sociales... inciden en la construcción de nuevas formas de identidad y nuevas formas de interpretar el mundo".

Por tanto, las relaciones personales y la identidad, se ven influidas en las interacciones presenciales y virtuales. No hay duda de que personas y grupos reducen cada vez más sus intereses y su quehacer cotidiano, a pequeños claustros de intercambio cibernético. Por consiguiente, la tecnología interviene en los individuos, en especial en los niños, niñas y adolescentes en el desarrollo de la personalidad, así como en: la interacción, comunicación, participación, autonomía, autoestima y en el ámbito socio-relacional.

Al respecto, son diversos los estudios que aportan una mirada sobre la comprensión del uso de las TIC, especialmente, en el entorno escolar y su incidencia en el ámbito socio-relacional, que se enfocan en dos direcciones: la primera, ligada directamente con lo académico y el uso que el entorno educativo da a la interacción social (Solano-Fernández, González-Calatayud \& López-Vicent, 2013); así como, la adopción y uso de medios sociales por jóvenes (López-Ponce \& Arcila-Calderón, 2016), aparte de formar en la apropiación segura de las TIC (Rueda-Rueda \& Rico-Bautista, 2016). La segunda, se preocupa por su incidencia en diversos ámbitos de los adolescentes para comprender procesos de socialización, a través de las Tecnologías de Información y Comunicación (TIC) (Pérez-Sierra, Hincapié-Marín \& Arias-Cardona, 2018), así como por las interacciones influidas por las TIC (Martínez-Baquero, 2016) y la incidencia de Facebook en las interacciones comunicativas de los adolescentes (Peralta-Idrovo \& Gallo-Orbe, 2012).

Lo anterior evidencia la importancia de las TIC y las relaciones sociales que de ellas emergen, 
como medio socio-relacional para el desarrollo de procesos pedagógicos, porque ofrecen posibilidades para la interacción, tanto con materiales didácticos en distintos formatos, como en la acción formativa en sí misma. Aunque los estudiantes en sus interacciones pueden tener relaciones sociales, así como vínculos que desarrollan: "relaciones entre actores y de las estructuras sociales que surgen de la recurrencia de esas relaciones" (Porras-Martínez, 2014), y la familiaridad con el uso de la red, esta a su vez presenta potencialidades y contradicciones.

En consecuencia, las TIC en el ámbito socio-relacional en los adolescentes, podría afectar lo pedagógico: "(...) se trata de una transformación en los procesos de producción y recepción de informaciones, cuyo sentido más general y cuyas consecuencias en la socialización de los niños, adolescentes y jóvenes, se desconocen y generan incertidumbre y preocupación" (Bernete-García, 2010).

En vista de la problemática señalada anteriormente, cobra sentido el identificar los nexos socio-relacionales del uso de las TIC en el entorno escolar de los adolescentes. Para ello, se utilizó el mapa socio-relacional, como instrumento de caracterización de un grupo de estudiantes de la educación media. A continuación, se presenta un breve marco teórico y se describe la metodología de la investigación. Luego, se describen los resultados y se brindan las conclusiones del estudio.

\section{Marco teórico y metodología}

2.1 Implicaciones socio-relacionales del uso de las TIC en adolescentes en el contexto escolar

En Colombia, las TIC se definen, como: "el conjunto de recursos, herramientas, equipos, programas informáticos, aplicaciones, redes y medios, que permiten: la compilación, procesamiento, almacenamiento, transmisión de información, como: voz, datos, texto, vídeo e imágenes" (Ley 1341, 2009). En este sentido, las TIC en el ámbito educativo, corresponden a un conjunto de diversas herramientas para: comunicarse, crear, difundir, almacenar y administrar información. Además, en determinados contextos, las TIC se han convertido en parte integral de la interacción enseñanza y aprendizaje.

Según la UNESCO (2008), para el uso de las TIC en el contexto escolar se requiere de nociones básicas, a través de las cuales se espera "incrementar la comprensión tecnológica de estudiantes, ciudadanos y fuerza laboral, mediante la integración de competencias en TIC en los planes de estudios -currículos-". Esta organización indica que las actividades que los estudiantes pueden realizar, conciernen al: uso de computadores y de software de productividad; entrenamiento, práctica, tutoría y contenido web; así como a la utilización de redes, con fines de gestión. Bajo esta perspectiva, las incidencias de las TIC en el medio escolar, algunas veces están determinadas por la comprensión de nociones básicas y su uso, así como por las interacciones en las redes sociales.

Ante este reto, las habilidades para el aprendizaje con el uso de "...las TIC, representan un nuevo contexto o ambiente donde los estudiantes se relacionan y vinculan con otros" (Centro de Educación y Tecnología, 2013). Es decir, las TIC en el medio escolar no solo forjan las habilidades de los adolescentes hacia al acceso a Internet en la búsqueda de información como una noción básica; sino que incluyen el uso de redes sociales y gestión de redes personales, lo cual tendrá incidencias en el ámbito socio-relacional de los estudiantes, como medio de interacciones socializadoras.

La interacción social se constituye cuando "dos o más individuos se encuentran en presencia de sus respuestas físicas respectivas, donde se involucran el estado de ánimo, la emoción, la cognición, la orientación corporal... los cuales suponen un elemento tanto biológico como psicológico" (Mercado-Maldonado \& Zaragoza-Contreras, 2011); sin embargo, "Ios estudiantes contemporáneos abandonan cada día la escuela 
y se introducen en un escenario de aprendizaje organizado de forma radicalmente diferente" (Pérez-Gómez, 2013).

Parece evidente que las TIC en su papel como medio de comunicación y de socialización en la educación, buscan mejorar procesos. En ese sentido, proporcionan un medio determinante en la formación de los adolescentes, que dan significado a las identidades establecidas en las relaciones sociales, puesto que hace necesario que un individuo conozca, adquiera y desarrolle formas de pensar, actuar y sentir, según normas de socialización en el contexto virtual. Las TIC permiten a los sujetos un medio para la relación con otros $y$, por consiguiente, dan lugar a un conjunto de diferentes emociones, comunicación y vínculos, así como a un proceso de "interacción social" (Kemper, 1978). Esto debido a que se han convertido en un elemento fundamental de cambio, en aspectos cotidianos del día a día (Colás-Bravo, de Pablos-Pons \& Ballesta-Pagán, 2018).

Lo anterior evidencia que las interacciones y relaciones entre los individuos, tienen un nuevo campo donde formarse, desarrollarse y evolucionar, a través de los espacios abiertos por Internet y el móvil, en las redes sociales. Este fenómeno afecta profundamente a las relaciones entre los individuos (Bernete-García, 2010), razón por la que podrían generarse innumerables vínculos en el intercambio personal, desembocando en un medio socializador y de interacción, basado en el uso exclusivo de las TIC, cuando haya la necesidad de interactuar con los demás.

En esta perspectiva, la teoría socio-relacional (Kemper, 1978), expone el poder y el estatus, como dimensiones de sociabilidad básicas, y es en ellas donde se forma un entramado de vínculos, en el que se trata de satisfacer y responder a las necesidades de un sujeto con la mediación del otro. Esta experiencia relacional, a su vez, lo que crea es una sensación positiva o negativa en el medio de las TIC. Además, Pérez-Salazar y Aguilar-Edwards (2012), mencionan que lo socio-relacional: es el asunto central de la teoría de redes sociales, que se expresa de muy diversas formas en los usuarios y las interacciones que se establecen. Esto a partir de la mediación tecnológica en la construcción de actos comunicativos y sociales, de vínculos frecuentes, capaces de establecerse como fuente para sus usuarios.

Las relaciones sociales mediante $\mathrm{TIC}$, según Sabater-Fernández, Martínez-Lorea y Campión (2017), generan una tecnosocialidad, ya que en las sociedades modernas las relaciones se pueden mantener tanto locales como de larga distancia, e influyen en el tipo e intensidad de las relaciones personales directas, que tienen hoy en día los jóvenes. Además, todas aquellas interacciones que fomentan la creación de una dinámica grupal, promueven las relaciones sociales y permiten expresar emociones (de Aguinaga-Vázquez, Ávila-González \& Barragán-de Anda, 2009). En este sentido, es importante comprender el entorno social establecido por la sociedad de la información y del conocimiento, así como el de las TIC como medio socio-relacional de los individuos, y las formas en que contribuyen a las conexiones sociales en la educación.

El uso de las TIC por parte de los educandos, se ha propagado al Internet, a través de: correo electrónico, chat, programación, ordenadores gráficos, ofimática en línea y búsqueda de información, entre otros. Martínez-González (2017), sostiene que en el medio de esta realidad se producen una serie de interacciones sociales mediadas por equipos que llegan, incluso, a condicionar las maneras en que los individuos conciben y estructuran, las relaciones interpersonales. Al respecto, Presutti (2012), sostiene que en el medio escolar, se debe realizar un "análisis de las relaciones psico-sociales". Asimismo, aduce que se deben visualizar o identificar: los espacios, los roles y los grupos de la clase. Porque las TIC y la Internet, como un artefacto relacional entre los jóvenes, permiten saber de otros y a la vez hacernos saber a otros (Gil-Juárez, Samuel-Lajeunesse, Espinosa-Zepeda \& Ramírez-Corvera, 2007). 
Pareciera que en este contexto escolar, se busca que los estudiantes establezcan nodos de conexiones. Es decir, el conocimiento se crea más allá del nivel individual, por lo que se conforman nodos de conectividad en una red, que da lugar a nuevas formas de conocimiento (Siemens, 2004; Gómez-Valderrama, Hernández-Suárez \& Prada-Núñez, 2020). Esta conectividad, según Echeverría-Ezponda (1999), se encuentra en el tercer entorno o en el entorno digital, en el que los individuos están estructuralmente distintos al E1 (primer entorno, el natural), y al E2 (segundo entorno, el urbano). En los contextos presencial y virtual de los estudiantes, los espacios que sirven para: la tarea, el ocio, la diversión, información personal, la influencia y las relaciones interpersonales, se pueden analizar con los mapas de interacción social (Martínez-Sánchez, Solano-Fernández \& Amat-Muñoz, 2012).

Las relaciones en el medio escolar, constituyen dinámicas de actividades en diferentes espacios (entornos), de interacción, en los cuales los estudiantes pueden corresponderse unos con otros y amplían la interacción conectiva en su ámbito de costumbre natural, incluso, esta interacción conectiva se puede ampliar con la tecnología (Presutti, 2012). En tal sentido, los sujetos están socio-relacionados; sin embargo, los adolescentes en el contexto escolar utilizan las TIC para fines generales, tales como: comunicación, procesamiento de textos y entretenimiento, en lugar de un único propósito educativo, conectados en un ecosistema comunicativo y relacional (Marta-Lazo \& Gabelas-Baroso, 2016). Las TIC se entienden como un medio donde coexisten: "un conjunto de nodos que forman redes sociales relacionales, y que dan lugar al surgimiento de estructuras que están basadas en los roles que los sujetos representan en su vida cotidiana" (Pérez-Salazar \& Aguilar-Edwards, 2012).

Según Martín-Barbero (2009), las TIC construyeron un nuevo ecosistema comunicativo en donde surgieron otros modos de producción de conocimiento, sustentados en una relación entre lo sensible y lo inteligible, donde las mediaciones tecnológicas evidencian un potencial alternativo. En consecuencia, muchos saberes socialmente valiosos circulan fuera de la escuela, sin pedirle permiso a la institución escolar.

\subsection{Método}

En esta investigación, se aplicó una metodología mixta, entendiendo que la producción de conocimiento es posible con la realización de estudios multimétodos, como: "una práctica para la integración de las perspectivas cuantitativa y cualitativa, expresada a través de: la combinación, la triangulación o la complementación desde los métodos" (Blanco \& Pirela, 2016). Además, es un estudio descriptivo, de corte transversal, que se adelantó en tres fases: diseño de la investigación, recolección de información, análisis y conclusión.

La población estuvo constituida por 800 estudiantes de la Institución Educativa Sin Fronteras, Cúcuta, Norte de Santander. En la muestra participaron 40 estudiantes, de los grados 9 a 11 , con edades que oscilan entre 14 y 17 años, con proporciones iguales de género. Del total de estudiantes, 34 completaron la encuesta, mientras que 14 participaron voluntariamente para la realización del mapa socio-relacional (Flórez-Camacho \& Corzo-Lindarte, 2018). La indagación se realizó con la técnica de construcción del mapa socio-relacional, para analizar las TIC como medio socializador. Además, se aplicó una encuesta enfocada en las actividades realizadas por los estudiantes a través de las TIC.

El mapa socio-relacional, es una técnica que tiene sus bases teóricas en el sociograma creado por Moreno (1954), y los mapas de interacción social (Martínez-Sánchez, Solano-Fernández \& AmatMuñoz, 2012), en el cual el comportamiento individual y grupal puede sustentarse en la interacción o el aislamiento de las relaciones sociales. En este caso, la finalidad fue determinar las relaciones y las características particulares del sujeto en su círculo social, tanto en el ámbito virtual como 
en el presencial. La encuesta tuvo un total de 22 ítems, orientados a las actividades efectuadas por medio de las TIC, así como a: el tiempo dedicado a estar conectado, redes sociales más significativas, impacto, repercusiones y beneficios académicos y sociales.

En la recolección de información, se preparó un mapa guía y preguntas orientadoras para que los estudiantes pudieran individualmente graficar sus interacciones. Para la prueba final, se utilizó un formato más práctico, en el que no se tenía que graficar, sino identificar las interacciones en los dos ámbitos, con las mismas preguntas orientadoras iniciales. En este caso, cada participante debía plasmar tres personas con las que interactuaba en situaciones determinadas, en los contextos presencial y virtual. El formato contenía 15 preguntas, referidas a: realización de tareas, diversión, salidas, interacción, influencia y el trato de temas personales.

El análisis de esta técnica, se hizo con base en el trabajo de Martínez-Sánchez, Solano-Fernández y Amat-Muñoz (2012), a través de los siguientes modelos: i) Modelo de costumbres; ii) Modelo de costumbres tecnológicamente ampliado; y iii) Modelo Tecnológico, en el cual se brindan las bases para realizar una matriz de relaciones por cada participante, en la que se vincula tanto la pregunta como las personas con las que interactúa, asignando un código a cada una de las interacciones que el participante señala. En esta investigación se adaptaron dichos modelos, donde se presenta la inicial del nombre de la persona, es decir, la inicial A corresponde al nombre de un estudiante, de lo contrario se utilizaba las iniciales de los dos nombres por ejemplo AN, mientras que al representante (Madre) se le asignó MA. Al final, se realizó una tabla con la información de dichas interacciones y las incidencias que había en los dos ámbitos.

Los mapas finales de cada participante, 14 en total, se analizaron a la luz de las teorías en conjunto e individualmente, para encontrar los patrones que vislumbran los posibles hallazgos de los procesos inter-relacionales de los adolescentes, en el contexto presencial y virtual, en los ámbitos: académico, ocio y personal. Lo académico se relaciona con el tema tarea, el ocio a diversión y salidas (cine u otro), y lo personal se sujeta a las relaciones, influencia y e información personal (ver tablas 1 y 2 ).

Tabla 1. Interpretación de las siglas de la tabla general de relaciones.

\begin{tabular}{llllllll}
\hline A & Abigail & $\mathrm{H}$ & Haylin & $\mathrm{MA}$ & Madre & $\mathrm{SE}$ & Sebastian \\
AN & Ana & $\mathrm{J}$ & Jaider & $\mathrm{MN}$ & Manuel & $\mathrm{SL}$ & Shirly \\
AL & Alejandra & JE & Jenny & $\mathrm{N}$ & Nora & $\mathrm{TA}$ & Tatiana \\
B & Brayan & $\mathrm{K}$ & Kevin & $\mathrm{NI}$ & Nicolás & $\mathrm{V}$ & Vanes \\
C & Camila & JP & Juan pablo & $\mathrm{O}$ & Omar & Y & Yesid \\
D & Daniela & KE & Kevin & P & Patricia & L & Lucho \\
DA & Danna & KA & Karla & PA & Paola & CH & Checo \\
ES & Esteban & CA & Carlos & RU & Rubén & & \\
G & Gabriel & M & María & S & Sofía & & \\
\hline
\end{tabular}


Tabla 2. Tabla general de relaciones.

\begin{tabular}{|c|c|c|c|c|c|c|c|c|}
\hline \multirow{2}{*}{$\begin{array}{l}\text { Ámbito } \\
\text { Académico }\end{array}$} & \multirow{2}{*}{$\begin{array}{l}\text { Temas } \\
\text { Tareas }\end{array}$} & \multicolumn{3}{|c|}{ Contexto presencial } & \multicolumn{3}{|c|}{ Contexto virtual } & \multirow{2}{*}{$\begin{array}{l}\text { Pres-virt } \\
0\end{array}$} \\
\hline & & $\mathrm{V}$ & & & & & & \\
\hline \multirow{2}{*}{ Ocio } & Diversión & V & G & $\mathrm{L}$ & & & & 0 \\
\hline & Salidas & G & $\mathrm{K}$ & V & G & $M$ & V & 1 \\
\hline \multirow{4}{*}{ Personal } & \multirow{2}{*}{ Relaciones } & MA & PA & $\mathrm{H}$ & $P$ & $A$ & $\mathrm{~K}$ & 0 \\
\hline & & $\mathrm{H}$ & AN & VA & G & V & AL & 0 \\
\hline & Influencia & G & MA & $\mathrm{H}$ & & & & 0 \\
\hline & Inf. personal & $\mathrm{V}$ & $\mathrm{K}$ & G & $\mathrm{S}$ & Y & $\mathrm{V}$ & 1 \\
\hline
\end{tabular}

En la Tabla 2, se observa que: $\mathrm{V}, \mathrm{G}, \mathrm{K}$ y $\mathrm{H}$, son interacciones del sujeto participante con las cuales posee una relación, tanto en el ámbito virtual como en el presencial. El estudiante V, se encuentra en cada uno de los temas que corresponden al modelo del mapa socio-relacional, pero existen contactos con los cuales únicamente se relaciona presencialmente, como es el caso de: MA, AN, PA, VA y L. Igualmente, en el ámbito virtual, existen contactos únicamente de este medio, como: $\mathrm{M}, \mathrm{P}, \mathrm{S}$ e $\mathrm{Y}$, lo cual indica que este sujeto tiene un modelo de interacción ampliada, ya que se relaciona virtual o presencialmente con diferentes personas, por lo que sus contactos e interacciones no son los mismos, lo que lo hace tener un círculo social más amplio. Es necesario resaltar que temas personales no son tratados en el ámbito virtual con contactos no constantes.

Esta muestra de las interacciones de los participantes, determina que el mapa de interacciones no solo establece las relaciones que tienen los adolescentes, sino más importante aún: qué interacción se está presentando en el contexto escolar con el uso de las TIC. Esto concuerda con el planteamiento de Martínez-Sánchez et al. (2012), quienes indican que este procedimiento permite analizar las posibles coincidencias o divergencias en los distintos planos de comunicación o contextos (presencial o tecnológicos), estudiados. Asimismo, se concuerda con Presutti (2012), porque el mapa socio-relacional representa una fotografía de las relaciones sociales del individuo, lo cual aporta los elementos necesarios para estructurar la intervención educativa, tanto en el ámbito presencial como en el virtual.

\section{Resultados y discusión}

En cuanto a las TIC, se encontró que los adolescentes se relacionan especialmente con tres aspectos: el internet en un $53 \%$, las redes sociales en un $32 \%$, y el uso de teléfonos móviles en un $25 \%$. Estos resultados concuerdan con Colás-Bravo, de Pablos-Pons y Ballesta-Pagán (2018), en que las TIC, como medio socializador, se han convertido en un elemento fundamental de cambio en la cotidianidad, dada la constante accesibilidad que estas ofrecen.

En lo relativo a la interacción en redes sociales, se comprobó que el $70 \%$ de adolescentes pasa 5 o más horas en medios tecnológicos, mientras que el 30\% restante pasa menos de 4 horas. Esta es una gran cantidad de tiempo, pues corresponde aproximadamente al $40 \%$ del día. Asimismo, el 80 por ciento de los participantes afirmó que la red social con la que más interactúa es Whatsapp, seguida por Facebook e Instagram, en las cuales casi el $50 \%$ dice hablar en ocasiones o constantemente, con personas desconocidas. Esto coincide con investigaciones que afirman que las TIC se han incorporado de manera habitual en la vida de los adolescentes, utilizándolas como herramientas de interacción, información, comunicación y conocimiento, especialmente, las redes sociales (Espinel-Rubio, Hernández-Suárez \& Rojas-Suárez, 2020). 
Respecto a la influencia de las TIC y los estados de ánimo de los adolescentes, se evidenció que el $65 \%$ manifiestan experimentar algún tipo de sentimiento negativo, cuando se les queda el teléfono; el más común es la rabia, seguido de la tristeza y la desmotivación. No obstante, un $35 \%$ dice no sentirse afectado por esta eventualidad, para su interacción social. Lo anterior coincide con Mercado-Maldonado y Zaragoza-Contreras (2011), para quienes la interacción social involucra: el estado de ánimo, la emoción, la cognición y la orientación corporal.

También se indagó sobre la preferencia en términos de salidas, donde se comparte personalmente fuera de la casa, tales como: fiestas, comer helado y hacer deporte, entre otras. El 62\% de los encuestados dice que en alguna ocasión o siempre, ha preferido quedarse en casa. Esta implicación de las TIC en la cotidianidad de los adolescentes, concuerda con Martínez-González (2017), quien sostiene que en medio de esta realidad, las TIC condicionan las maneras en que los individuos conciben y estructuran las relaciones interpersonales.

En el aspecto socio-relacional de los adolescentes de la institución educativa, se halló que depende de las características individuales de cada uno. En este sentido, se presenta un análisis descriptivo caracterizado por un número reducido de relaciones, ya que aquellos contactos con los que se interactúa en el ámbito presencial son los mismos del ámbito virtual. La figura 1, adaptada de Martínez-Sánchez et al. (2012), muestra la representación gráfica de las interacciones que se disponen en la Tabla 3.

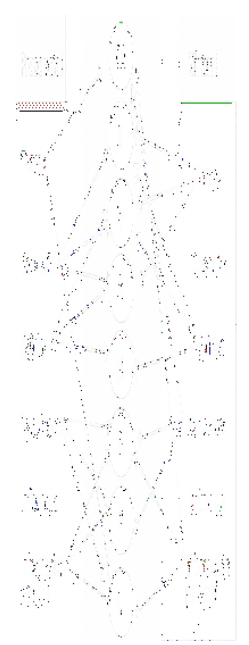

Figura 1. Mapa socio relacional - modelo de interacción conectiva.

En la figura 1, se percibe que cada uno de los contactos está presente en más de dos temas, en las columnas presencial y virtual, independientemente de la frecuencia y del número de interacciones en red. Las TIC son parte esencial del contacto con otros, tanto en el contexto virtual como en el presencial, por eso son conectivas, pues los medios sociales están presentes en los procesos de socialización de quienes hacen parte de este tipo de interacción. Es decir, el círculo social se centra en las mismas interacciones y la representación gráfica lo confirma. Esto coincide con Martínez-Sánchez et al. (2012), donde la persona cuenta siempre con la misma red de contactos para interaccionar en el plano presencial, en el móvil y en Internet, lo cual refleja el modelo de costumbres. 
Tabla 3. Relaciones interacción conectiva.

\begin{tabular}{lllllllll}
\hline Ámbito & Temas & \multicolumn{2}{l}{ Contexto presencial } & \multicolumn{2}{l}{ Contexto virtual } & & Pre-vir \\
\hline Escolar & Tareas & CA & L & & $\mathrm{K}$ & $\mathrm{A}$ & $\mathrm{V}$ & 0 \\
\multirow{2}{*}{ Ocio } & Diversión & $\mathrm{K}$ & $\mathrm{A}$ & $\mathrm{V}$ & & & & 0 \\
& Salidas & $\mathrm{V}$ & $\mathrm{A}$ & $\mathrm{H}$ & $\mathrm{K}$ & $\mathrm{V}$ & $\mathrm{H}$ & 2 \\
& Relaciones & $\mathrm{CA}$ & $\mathrm{B}$ & $\mathrm{P}$ & $\mathrm{L}$ & $\mathrm{P}$ & $\mathrm{B}$ & 1 \\
& Influencia & $\mathrm{K}$ & $\mathrm{CA}$ & $\mathrm{L}$ & $\mathrm{K}$ & $\mathrm{P}$ & $\mathrm{L}$ & 2 \\
\multirow{3}{*}{ Personal } & Inf. Personal & $\mathrm{CA}$ & $\mathrm{L}$ & $\mathrm{B}$ & $\mathrm{L}$ & $\mathrm{B}$ & $\mathrm{P}$ & 2 \\
\hline
\end{tabular}

En la Tabla 4, se presentan las interacciones de un grupo reducido de adolescentes. Asimismo, se representa el mapa socio relacional del modelo de conectividad ampliada (figura 2), y el mapa de interacciones de algunos estudiantes con respecto al ámbito escolar y personal (figura 3).

Tabla 4. Relaciones conectividad ampliada de un grupo de participantes.

\begin{tabular}{|c|c|c|c|c|c|c|c|c|}
\hline \multirow{2}{*}{$\begin{array}{l}\text { Ámbito } \\
\text { Escolar }\end{array}$} & \multirow{2}{*}{$\begin{array}{l}\text { Temas } \\
\text { Tareas }\end{array}$} & \multicolumn{3}{|c|}{ Contexto presencial } & \multicolumn{3}{|c|}{ Contexto virtual } & \multirow{2}{*}{$\begin{array}{l}\text { Pre-vir } \\
0\end{array}$} \\
\hline & & L & $\mathrm{MN}$ & $\mathrm{D}$ & RO & M & JN & \\
\hline \multirow{2}{*}{ Ocio } & Diversión & SL & DS & A & & & & 0 \\
\hline & Salidas & $C A$ & $\mathrm{D}$ & $A L$ & MG & $\mathrm{Cl}$ & LU & 0 \\
\hline \multirow{4}{*}{ Personal } & \multirow{2}{*}{ Relaciones } & L & $\mathrm{MN}$ & SY & $\mathrm{AL}$ & 0 & $\mathrm{D}$ & 0 \\
\hline & & B & BY & RO & SL & VS & $C A$ & 0 \\
\hline & Influencia & MN & $\mathrm{L}$ & RO & & & & 0 \\
\hline & Inf. Personal & L & BY & LU & $\mathrm{AL}$ & $\mathrm{P}$ & L & 1 \\
\hline
\end{tabular}

Figura 2. Mapa socio relacional, Modelo de conectividad ampliada para un grupo de adolescentes. 
En la figura 2, se puede percibir que algunos contactos únicamente se encuentran en el entorno virtual, lo cual hace que el círculo social de dicho sujeto sea más amplio; sin embargo, cabe resaltar que algunas interacciones presenciales también se encuentran dispuestas en el ámbito virtual, en temas relacionados con: las tareas, relaciones e información personal. Otra característica importante, es que los contactos que solo pertenecen al ámbito virtual, tienen un tema específico de interacción; es decir, no se relacionan en más de dos aspectos de la vida del sujeto. Esto se debe a que el número de relaciones que tienen los adolescentes en el medio virtual, son diferentes a las del medio presencial (Marta-Lazo \& Gabelas-Baroso, 2016). Ello gracias a que existe mayor conectividad comunicativa; los adolescentes no mencionan ningún contacto en el medio virtual, que sea influyente en su vida y sus decisiones.

En el modelo de costumbres tecnológicamente ampliado, el medio tecnológico sirve como mecanismo de socialización y apoyo de estas amistades, ya que estos individuos forman parte de la vida virtual del mismo modo que los de la presencial (Martínez-Sánchez et al., 2012). Así se reafirma que las TIC: "no son un medio de aislamiento social, ya que la red puede funcionar como un medio de socialización básico de los estudiantes" (Solano-Fernández, González-Calatayud \& López-Vicent, 2013). Es decir, estas se convierten en un medio para la interacción, donde se puede compartir de formas diferentes en los aspectos: educativo, personal y el ocio. Para los adolescentes son un espacio "libre de restricciones, en el que se sienten libres y pueden buscar su autenticidad, a través de: juegos, relaciones, conversaciones, es decir, un marco más general del conocimiento de la sociedad" (Peralta-Idrovo \& Gallo-Orbe, 2012).

Cuando se habla de implicaciones, generalmente se asocian a aspectos negativos. En este estudio, se trataron de determinar esas incidencias en las relaciones humanas de los adolescentes. Se encontró que los usos responden a la representación conceptual que tienen los adolescentes de las TIC, que son un ente socializador indiscutible. Aunque son innegables las ventajas que lo presencial brinda a las interacciones, especialmente en la percepción y las emociones, se comprobó que las TIC tienen un efecto capaz de transformar conductas e influir en la toma de decisiones de esta población. 


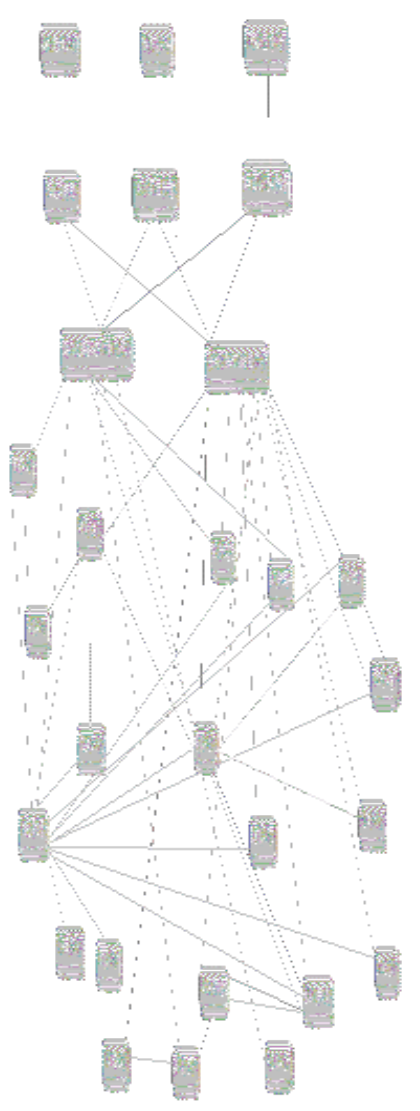

Figura 3. Mapa de interacciones de los estudiantes, respecto a los ámbitos: escolar, ocio y personal.

En la figura 3, se muestra que la interacción fluye entre: estudiante-estudiante; estudiante-representante y estudiante con algunos sujetos externos al medio escolar. Se observa que los pares ni siquiera necesitan realimentar para relacionarse socialmente con otros pares y sujetos, para compartir en su pequeño grupo. Igualmente, se determinó que no todos los estudiantes tienden a tener una conexión socio-relacional con sus compañeros de clase en lo virtual, a pesar de compartir en el ambiente escolar de manera presencial. Asimismo, se muestra un proceso colectivo de producción de conocimiento sobre las tareas, tanto en el contexto presencial como en el virtual. En este sentido, las TIC juegan un papel importante en el conjunto de fenómenos autorreguladores en las relaciones sociales.
Se infiere que las interacciones mostradas, revelan una representación de interacciones conectivas, las cuales son ampliadas por los diversos recursos tecnológicos en el ámbito educativo, ocio y personal, de cada estudiante. Más allá de su papel como medio socializador, los educandos aplican las TIC directa o indirectamente, a los contextos que unen y motivan a los adolescentes en diferentes tipos de interacciones socio-relacionales, dentro o fuera del medio escolar. Es decir, se desarrollan nuevas formas de producción de conocimiento, mediadas por la tecnicidad y descentralizadas (Martín- Barbero, 2009).

\section{Conclusiones}

Los adolescentes emplean herramientas tecnológicas de acuerdo con la apreciación que tienen 
sobre ellas; es decir, como medio socio-relacional, más que por el flujo de información y gestión del conocimiento que se presenta en el ámbito escolar; sin embargo, la constante presencia en la red, ha transformado las estrategias de relacionamiento y aprehensión de conocimiento. Las redes sociales que se configuran en lo virtual, les permiten obtener información que es de difícil acceso en la presencialidad.

El mapa socio-relacional, como instrumento de recolección de información, permitió identificar las interacciones de dos formas: el conectivo de costumbre y/o conectivo ampliado. El primero, se caracteriza por aquellas conexiones de costumbres que se encuentran en el ámbito presencial, son las mismas del ámbito virtual. En el segundo, los adolescentes tienen mayor conectividad comunicativa con otros individuos, distintos a los sujetos que se encuentran en el ámbito presencial y virtual de costumbre.

Las TIC como medio socio-relacional en adolescentes en el contexto escolar, registran un proceso de relaciones e interacciones, dadas a partir de: la comunicación, lenguaje y significados compartidos entre los sujetos. Estas contribuyen a la construcción de sus realidades inter-relacionales, que posibilitan la socialización de experiencias de aprendizaje, así como de información y otros elementos que podrían enriquecer el proceso de enseñanza y de aprendizaje, en el contexto escolar.

La interacción de los estudiantes en la red virtual, está determinada por los diversos equipos tecnológicos y aplicaciones, que convierten a las TIC en escenarios para la formación social, lo que se refleja en una menor o mayor comunicación e información con respecto a su conectividad. No obstante, ante esta realidad, también se disminuye el número de sus interacciones presenciales, lo que puede llegar a afectar las sensaciones de veracidad y el hecho de percibir lo que es real.

En este sentido, las TIC se han convertido en un medio primordial para el proceso de socialización de los adolescentes, así como para ampliar su capacidad y habilidades sociales y relacionales, para: exteriorizar ideas, emociones, aspiraciones y opiniones de forma adecuada, directa, equilibrada, honesta y respetuosa. Esto, sin el propósito de inquietar al otro con el mensaje emitido, que conlleva una serie de elementos, tales como: intencionalidad, entonación y cordialidad, que sin duda, afectan e inciden en la comunicación.

\section{Referencias}

Bernete-García, F. (2010). Usos de las TIC, Relaciones sociales y cambios en la socialización de las y los jóvenes. Revista de Estudios de Juventud, 88, 97-114. Recuperado de: http://www.injuve.es/ sites/default/files/RJ88-08.pdf

Blanco, N., \& Pirela, J. (2016). La complementariedad metodológica: Estrategia de integración de enfoques en la investigación social. Espacios públicos, 19 (45).

Carrillo-David, S. (2015). Infancia y socialización mediática: el papel de las tecnologías de la información y la comunicación (TIC) en la socialización de un grupo de niños y niñas de 5 y 6 años de edad (Tesis de Maestría). Universidad de Antioquia, Medellín. Recuperado de: http://hdl.handle.net/10495/6485

Centro de Educación y Tecnología, Enlaces. (2013). Matriz de Habilidades TIC para el Aprendizaje. Santiago de Chile: Ministerio de Educación.

Colás-Bravo, M., de Pablos-Pons, J., \& Ballesta-Pagán, J. (2018). Incidencia de las TIC en la enseñanza en el sistema educativo español: una revisión de la investigación. Revista de Educación a Distancia, 18 (56), 1-23. doi: http://dx.doi. org/10.6018/red/56/2

De Aguinaga-Vázquez, P., Ávila-González, C., \& Barragán de Anda, A. (2009). Presencia social, didáctica y cognitiva del docente a distancia. Apertura. 1 (1), 66-75. Recuperado de: http://www. 
udgvirtual.udg.mx/apertura/index.php/apertura/ article/view/115

Echeverría-Ezponda, J. (1999). Los señores del aire: Telepolis y el tercer entorno. Barcelona: Destino.

Espinel-Rubio, G., Hernández-Suárez, C., \& RojasSuárez, J. (2020). Usos, apropiaciones y nuevas prácticas comunicativas de los usuarios adolescentes de Facebook. Saber, Ciencia y Libertad, 15 (1), 280-296. doi: https://doi.org/10.18041/2382-3240/ saber.2020v15n1.6316

Flórez-Camacho, M., \& Corzo-Lindarte, E. (2018). Implicación socio relacional del uso de las TICs en el contexto escolar en adolescentes de grados $9^{\circ}, 10^{\circ} \mathrm{y}$ $11^{\circ}$ del colegio Sin Fronteras de Cúcuta (Trabajo de grado). Universidad Francisco de Paula Santander, Cúcuta, Colombia. Recuperado de: http://alejandria.ufps.edu.co/descargas/tesis/1340342.pdf

Gil-Juárez, A., Samuel-Lajeunesse, J., Espinosa-Zepeda, H., \& Ramírez-Corvera, M. (2007). Internet como un artefacto relacional: jóvenes, migraciones y TIC. En Psicología Social: Un Encuentro de Perspectivas, 492-497, Cádiz: Asociación de Profesionales de Psicología.

Gómez-Valderrama, C., Hernández-Suárez, C., \& Prada-Núñez, R. (2020). La zona de posibilidades en el proceso de aprendiencia del residente digital: un análisis cualitativo en la red de experiencias matemáticas de Norte de Santander. Educación y Humanismo, 22 (38), 1-19. doi: http://dx.doi. org/10.17081/eduhum.22.38.3688

Islas-Torres, C. (2017). La implicación de las TIC en la educación: Alcances, limitaciones y prospectiva. RIDE Revista Iberoamericana para la Investigación y el Desarrollo Educativo, 8 (15), 861-876. doi: https:// doi.org/10.23913/ride.v8i15.324

Kemper, T. (1978). A Social Interactional Theory of Emotions. New York: Wiley.
Ley 1341 (30 de julio de 2009). Congreso de Colombia, Bogotá, Colombia.

López-Ponce, M., \& Arcila-Calderón, C. (2016). Adopción y uso de los medios sociales por jóvenes en la Costa Caribe de Colombia. Investigación \& Desarrollo, 24 (2), 285-307. doi: http://dx.doi. org/10.14482/indes.24.2.8906

Marta-Lazo, C., \& Gabelas-Baroso, J. (2016). Comunicación Digital. Un modelo basado en el factor Relacional. Barcelona: Editorial UOC

Martín-Barbero, J. (2009). Cuando la tecnología deja de ser una ayuda didáctica para convertirse en mediación cultural. Education in the Knowledge Society (EKS), 10 (1), 19-31. doi: http://dx.doi. org/10.14201/eks.15549

Martínez-Baquero, M. (2016). Interacciones influidas por el uso de TIC, en familias con hijos adolescentes en la ciudad de Bogotá (trabajo de grado). Universidad Externado de Colombia, Bogotá, Colombia. Recuperado de: https://bdigital.uexternado.edu. co/handle/001/349

Martínez-González, Y. (2017) De las TIC a las TRIC. Una nueva realidad socio-comunicacional en Cuba. Index Comunicación, 7 (3), 187-209. Recuperado de: https://journals.sfu.ca/indexcomunicacion/index.php/indexcomunicacion/article/ view/319

Martínez-Sánchez, F., Solano-Fernández, I., \& Amat-Muñoz, L. (2012). Análisis de mapas de interacción social en contextos virtuales para la reinterpretación de las relaciones en la escuela. Revista Latinoamericana de Tecnología Educativa RELATEC, 11 (1), 13-26. Recuperado de: https://relatec.unex. es/article/view/849

Mercado-Maldonado, A., \& Zaragoza-Contreras, L. (2011). La interacción social en el pensamiento sociológico de Erving Goffman. Espacios Públicos, 
14 (31), 158-175. Recuperado de: https://www. redalyc.org/articulo.oa?id=676/67621192009

Moreno, J. (1954). Fundamentos de la Sociometría. Buenos Aires: Paidós.

Peralta-Idrovo, L., \& Gallo-Orbe, L. (2012). La influencia de facebook en las interacciones comunicativas de los adolescentes de 15-16 años del primer año de bachillerato (trabajo de grado). Universidad Central del Ecuador, Quito, Ecuador.

Pérez-Gómez, Á. (2013). Educarse en la era digital: Adelanto del nuevo libro de Ángel Pérez Gómez. Sinéctica, 40, 01-26. Recuperado de: https:// sinectica.iteso.mx/index.php/SINECTICA/article/ view/51

Pérez-Salazar, G., \& Aguilar-Edwards, A. (2012). Reflexiones conceptuales en torno a las redes sociales en las redes sociales: un recorrido de la teoría a las prácticas comunicativas en Facebook, Twitter y Google+. Razón y Palabra, 79. Recuperado de: http://www.razonypalabra.org.mx/N/ N79/V79/06_PerezAguilar_V79.pdf

Pérez-Sierra, M., Hincapié-Marín, B., \& Arias-Cardona, A. (2018). Socialización de jóvenes a través de las TIC en una institución educativa de Antioquia. Pensamiento psicológico, 16 (2), 59-72. Recuperado de: https://revistas.javerianacali.edu. co/index.php/pensamientopsicologico/article/ view/1683

Porras-Martínez, J. (2014). Internet y Comunidad: Una aproximación desde el enfoque del estudio de redes personales. Polis, Revista Latinoamericana,
13 (39), 203-226. doi: http://dx.doi.org/10.4067/ S0718-65682014000300010

Presutti, F. (2012). La formación docente en la Educación de Infancia: El desarrollo de las capacitaciones profesionales para la realización del CIF - Centro de Infancia y de la Familia. Editorial Académica Española.

Rueda-Rueda, J., \& Rico-Bautista, D. (2016). Inseguridad en las redes sociales e internet: formando en la apropiación segura de las TIC. Revista Colombiana de Computación, 17 (1), 55-72. doi: http:// dx.doi.org/10.29375/25392115.2625

Sabater-Fernández, C., Martínez-Lorea, I., \& Campión, R. (2017). La Tecnosocialidad: El papel de las TIC en las relaciones sociales. Revista Latina de Comunicación Social, 72, 192-1607. doi: http:// dx.doi.org/10.4185/RLCS-2017-1236

Siemens, G. (2004). Connectivism: A learning theory for the digital age. International Journal of Instructional Technology and Distance Learning, 2 (1), 3-10. Recuperado de: http://citeseerx.ist.psu. edu/viewdoc/download?doi=10.1.1.87.3793\&re$\mathrm{p}=$ rep $1 \&$ type $=$ pdf

Solano-Fernández, I., González-Calatayud, V., \& López-Vicent, P. (2013). Adolescentes y comunicación: las TIC como recurso para la interacción social en educación secundaria. Pixel-Bit. Revista de Medios y Educación, 0 (42), 23-35. Recuperado de: https://recyt.fecyt.es/index.php/pixel/article/ view/61566

UNESCO. (2008). Estándares de Competencias en TIC para Docentes. Londres: UNESCO. 